\title{
The Determinants of Fatal Outcomes during Severe Malaria in Children at the HKM University Teaching Hospital of Cotonou-Benin
}

\author{
Godonou Gratien Sagbo ${ }^{*}$, Florence Alihonou1, Marouf Jules Alao², Yévèdo Tohodjèdé1,

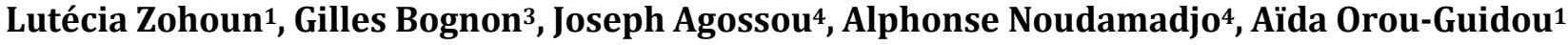 \\ ${ }^{1}$ Pediatric Unit, CNHU-HKM, Cotonou, Benin \\ ${ }^{2}$ Lagoon Mother and Child Hospital, Cotonou, Benin \\ ${ }^{3}$ Hospital of Porto-Novo, Porto-Novo, Benin \\ ${ }^{4}$ Pediatric Unit, CHU-Borgou, Parakou, Benin \\ Email: *godsagbo@yahoo.fr
}

How to cite this paper: Sagbo, G.G., Alihonou, F., Alao, M.J., Tohodjèdé, Y., Zohoun, L., Bognon, G., Agossou, J., Noudamadjo, A. and Orou-Guidou, A. (2017) The Determinants of Fatal Outcomes during Severe Malaria in Children at the HKM University Teaching Hospital of Cotonou-Benin. Open Journal of Pediatrics, 7, 245-253.

https://doi.org/10.4236/ojped.2017.74028

Received: September 21, 2017

Accepted: November 18, 2017

Published: November 21, 2017

Copyright (ㅇ 2017 by authors and Scientific Research Publishing Inc. This work is licensed under the Creative Commons Attribution International License (CC BY 4.0).

http://creativecommons.org/licenses/by/4.0/

\begin{abstract}
Introduction: Malaria particularly affects children in sub-Saharan African countries. This study aimed to investigate the factors associated with death in cases of severe childhood malaria to better understand the determinants of death in these children. Patients and Methods: This cohort, descriptive and analytical study was conducted from April 1 to August 15, 2015, at the CNHU-HKM pediatric clinic in Cotonou. Recruitment was exhaustive for all patients under 15 years of age who were admitted for severe malaria, as confirmed by thick smear microscopy. Results: Among the 1774 admitted patients, 449 had severe malaria caused by Plasmodium falciparum (i.e., a hospital frequency of $25.31 \%$ ). The age group most affected consisted of children younger than 60 months of age $(73 \%)$; female predominance was noted. The lethality rate of malaria was $13.1 \%(n=59)$. The factors associated with death were coma $(\mathrm{p}=0.032)$, poor convulsive status epilepticus $(\mathrm{p}=0.08)$ and bacterial co-infection by gram negative bacteria ( $\mathrm{p}=0.021$ ) with respectively correlations coefficient of $0.003,3.940$ and 2.424. Conclusion: Reduction of the malaria mortality rates in Benin hospitals will depend on appropriate management of poor prognostic factors, such as coma, bacterial co-infection and convulsive illness.
\end{abstract}

\section{Keywords}

Children, Severe Malaria, Lethality, Neurological Disorders, Bacterial Infection 


\section{Introduction}

Malaria is a major health issue worldwide, with more than 3.2 billion people affected, despite investments to prevent and control the disease [1]. According to the WHO, in 2013, severe malaria caused 584 thousand deaths per year worldwide, including $90 \%$ in Sub-Saharan Africa, and children younger than 5 years of age were the primary victims (78\%) [2]. In Benin, malaria mortality has demonstrated peaks and dips since 2000, with an average of $8.8 \%$ [3]. However, some progress has been made since 2000 , with a mortality rate that declined to $47 \%$ globally and to $54 \%$ in Africa [4]. These advances are related to several interventions, particularly the Roll Back Malaria (RBM) partnership, use of the malaria rapid diagnostic test (RDT) in all peripheral health facilities, and the distribution of kits to manage severe malaria in all hospitals, particularly in Benin. Despite that downward trend, the mortality rate is still high. Some authors have identified certain risk factors associated with death in severe malaria cases [5] [6]. Other factors might also explain why the trend is still high. The authors of this study aimed to identify the determinants of death in cases of severe malaria in children at the CNHU-HKM of Cotonou.

\section{Patients and Methods}

This cohort, descriptive and analytical study was performed from April 1 to August 15, 2015, and it focused on children from one month to 15 years of age who were hospitalized for severe malaria in the Pediatric Unit of the Hubert K. Maga National University Teaching Hospital of Cotonou. Authorization was obtained from the Faculty of Health Sciences' Ethical Committee in Cotonou and informed consent of patient's parents before starting the study. The researchers defined severe malaria as a thick smear test that was positive for Plasmodium falciparum, associated with one or many serious symptoms, according to the World Health Organization's recommendations [1]. This study did not enroll children who were suffering from severe malaria and were deceased upon admission, or whose illness was associated with sickle cell disease. Recruitment was exhaustive and based on child hospitalization records or on the register. The data were gathered using a data processing form. The study variables were data related to the children (i.e., age, sex, nutritional status, clinical and paraclinical serious symptoms of malaria, clinical and paraclinical signs of bacterial co-infection, length of hospital stay, therapeutic characteristics and children's future) and to the parents in charge of the children (age, educational background and occupation), as well as the health system, if the case was a referral (referring health center and waiting time for the referral).

The data (generated with full confidentiality) were processed and analyzed using SPSS 2.1 software and Pearson's Chi-squared test when the number of subjects under observation was greater than or equal to 5 , Yates corrected Chi-squared test when the number was between 5 and 2.5, and finally, the Fisher exact test when the number was lower than 2.5. Each time a relationship was established between occur- 
rence of death and one of the study variables, the level of the relationship was measured using an odds ratio (OR) with a $95 \%$ confidence interval. Any result was considered to be statistically significant when $p$ was lower than $5 \%$. Univariate analysis was complemented by multivariate analysis using binary logistic regression to identify the independent poor prognostic factors associated with the lethality of malaria.

\section{Results}

\subsection{Characteristics of the Study Target Population}

During the study, 449 children (of a total of 1774 admissions) were investigated (i.e., the frequency of hospital admissions due to severe malaria caused by Plasmodium falciparum was estimated at $25.31 \%)$. The sex ratio (M/F) was 0.9 with 212 males. Thirty children (6.7\%) were younger than 12 months of age, 296 (65.9\%) were 12 months to 60 months of age, and 123 children (27.4\%) were older than 60 months. The mean age of the children was 51 months (range, 2 to 180 months). Children younger than 5 years of age accounted for $72.6 \%$ (326/449) of the study's target population. The most common serious symptoms were anemia 279/449 (62.2\%), "Coca cola" urine 170/449 (37.9\%), and coma 152/449 (33.8\%) (Figure $1)$.

The average length of hospital stay was 5 days (range, 0 to 25 days). Fifty-nine of the 449 children involved in the study experienced fatal outcomes (i.e., a hospital-related lethality of severe malaria was estimated at 13.1\%; 95\% Confidence Interval: 10.0 - 16.3). Two of three children died within the first 24 hours of hospital admission.

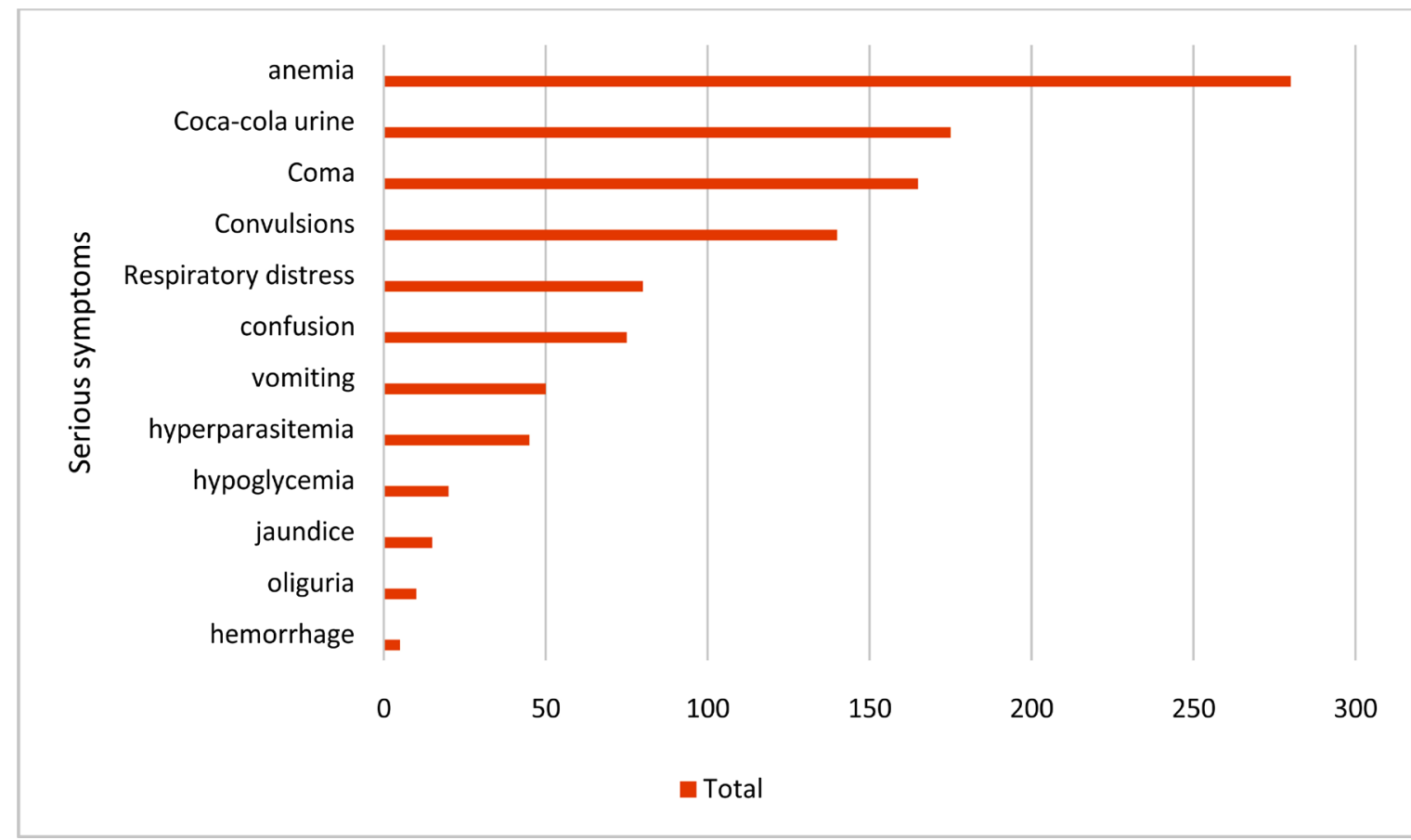

Figure 1. Distribution of children, according to symptoms of severe malaria. 


\subsection{Comparison of Children Hospitalized for Malaria and Lived Compared to Those Who Died}

\subsubsection{Epidemiological Characteristics of Children, Educational Backgrounds and Occupations of Their Guardians and Aspects of Referrals}

The children's epidemiological factors, the characteristics their guardian, and aspects of the referral system discussed in this study had no influence on the risk of death among the children hospitalized for malaria. Approximately three of four children $(329 / 449,71.2 \%)$ were referred by another health facility.

\subsubsection{Clinical Characteristics of the Children}

1) Nutritional status

Nutritional status was poor in 12 of the deceased children (of a total of 64 malnourished children), but it did not significantly increase the risk of death.

2) Signs of severity

Among the serious symptoms observed (Figure 1$)$, only coma $(\mathrm{p}<0001)$, convulsions $(\mathrm{p}=0.005)$, hypoglycemia $(\mathrm{p}=0.005)$ and respiratory distress $(\mathrm{p}<0.001)$ were associated with death (Table 1 , page 5 ).

3) Bacterial co-infection

Eighty-seven of the 449 children (19.4\%) presented with a bacterial co-infection; 19 of the 87 (21.8\%) died. The bacterial infections were septicemia, meningitis, urinary infection and enterocolitis. There was a relationship between the occurrence of death and bacterial co-infection, particularly septicemia $(p<0.001)$ (Table 2).

4) Therapeutic characteristics

The therapeutic characteristics examined were the wait time for care, use of artesunate, quinine, anticonvulsants and antibiotics, and length of hospital stay, as well as undergoing a blood transfusion. Regarding protective effects, there was a relationship between patient mortality and several therapeutic factors (protective factors): use of artesunate ( $\mathrm{p}<0.001)$, anticonvulsants $(\mathrm{p}<0.001)$, antibiotics $(\mathrm{p}=0.012)$ and blood transfusion $(\mathrm{p}=0.019)($ Table 3$)$.

\subsection{Factors Associated with Death}

After the logistic regression analysis, the constant factors associated with children's death were coma, bacterial co-infection and convulsive status epilepticus, with a 95\% confidence interval (Table 4).

\section{Discussion}

This study analyzed the characteristics and critical factors related to hospital-related lethality of severe malaria in children at CNHU-HKM. The most critical factors associated with those children's death were convulsive status epilepticus, coma and bacterial co-infection.

The limitations of the study lie in the difficult realization of complementary examinations because of they were expensive and the audit of deaths based on a verbal autopsy. The results obtained revealed significant correlations between malaria 
Table 1. Distribution of living or deceased children, according to serious symptoms of malaria.

\begin{tabular}{ccccc}
\hline & Death n/N (\%) & Total & QR & \\
\hline Coma & & & & \\
Yes & $39 / 152(25.7)$ & 152 & $4.78(2.67-8.55)$ & $<0.001$ \\
No & $20 / 297(6.7)$ & 297 & & 0.005 \\
Repeated convulsions & & & \\
Yes & $28 / 142(19.7)$ & 142 & $2.19(1.21-3.95)$ & \\
No & $31 / 307(10.1)$ & 307 & & \\
Respiratory distress & & & & \\
Yes & $22 / 77(28.6)$ & 77 & $3.62(1.99-6.60)$ & \\
No & $37 / 372(9.9)$ & 372 & & \\
Hypoglycemia & & & & \\
Yes & $7 / 18(38.9)$ & 18 & $4.64(1.72-12.49)$ & \\
No & $52 / 431(12.1)$ & 431 & & \\
\hline
\end{tabular}

Table 2. Distribution of living or deceased children, according to presence of bacterial co-infection.

\begin{tabular}{ccccc}
\hline & Death $\mathrm{n} / \mathrm{N}(\%)$ & Total & OR & P \\
\hline Septicemia & & & & \\
Yes & $11 / 19(61.1)$ & 19 & $10.31(2.7-39.98)$ & $<0.001$ \\
No & $8 / 68(11.8)$ & 68 & & \\
Pneumonia & & & & \\
Yes & $4 / 40(10.0)$ & 40 & $0.24(0.06-0.86)$ & 0.014 \\
No & $15 / 47(31.9)$ & 47 & & \\
\hline
\end{tabular}

Table 3. Distribution of living or deceased children, according to their therapeutic characteristics.

\begin{tabular}{ccccc}
\hline & Death & Total & OR & P \\
\hline $\begin{array}{c}\text { Artesunate } \\
\text { Yes }\end{array}$ & $50(11.4 \%)$ & 437 & & \\
No & $9(75.0 \%)$ & 12 & $23.22(6.08-88.63)$ & $<0.001$ \\
Antibiotic therapy & & & $2.03(1.12 ; 3.68)$ & 0.012 \\
Yes & $26(19.3 \%)$ & 135 & & \\
No & $33(10.5 \%)$ & 314 & & \\
Total & 59 & 449 & & \\
\hline
\end{tabular}

NOTE: the use of artesunate, anticonvulsants and antibiotics protects against death.

Table 4. Critical factors associated with occurrence of death $=$ specify multivariate analysis.

\begin{tabular}{ccccc}
\hline Variables & P & Coef. & \multicolumn{2}{c}{$95 \%$ CI } \\
\hline Coma & 0.032 & 0.003 & 1.098 & 8.216 \\
Co-infection & 0.021 & 2.424 & 1.166 & 4.080 \\
Convulsive status epilepticus & 0.008 & 3.940 & 1.426 & 10.884 \\
\hline
\end{tabular}


death and associated factors.

\subsection{Epidemiological Aspects}

The frequency of malaria in hospitalized children in this study was $25.3 \%$, which is close to the 29.8\% $(n=409)$ observed in Tanzania in 2012 [6]. In Togo in 2002 and in Senegal in 2007, rates of $4.37 \%(\mathrm{n}=361)$ and $6.4 \%(\mathrm{n}=162)$, respectively, were reported in hospitalized children. These figures are much lower than ours [7] [8]. Those differences may be due to many factors. First, this research was performed during the rainy season, which is characterized by more cases of malaria, whereas in Togo and Senegal, the study periods were longer (3 years and 1 year, respectively) and included periods of low transmission of severe malaria. In addition, the type of transmission is highly endemic in Benin, whereas in Senegal, a country with a Sudano-Sahelian climate, malaria transmission is intermittent (i.e., more common during the rainy season and low during the rest of the year) [9]. As a result, the CNHU facility that hosts the national blood transfusion center treats most children suffering from severe malaria for anemia $(62.5 \%$ of cases) because of a frequent shortage of blood products in the other hospitals of the area during times of high demand.

In this series, the mean age of the children was 52 months, and children younger than 5 years of age were the numerically predominant population (73\%). This age group was most affected by cases of severe malaria, as reported by another author [10]. The predominance of that age group may be due to the disappearance at that age of the innate immunity provided by the presence of fetal hemoglobin and food diversification. The latter is not always well conducted in our context due to poverty on the one hand and to the late acquisition of premunition against malaria on the other hand [11]. In Senegal, according to two studies conducted in 1999 and 2007, respectively, the predominant age group was that of children 5 to 10 years of age [8] [9]. According to the authors, it is one of the characteristics of malaria intermediary host areas, such as Senegal.

\subsection{Clinical Aspects}

The most common serious symptoms were (in descending order of occurrence) anemia (62.6\%), "Coca Cola" urine (38.1\%) and coma (34.1\%). Another author has already identified severe anemia as the most common serious symptom [12]. Its high frequency, in terms of iron deficiency was present in 30 to $60 \%$ of the children younger than 5 years of age in tropical Africa [13] [14], and as CNHU is the national referral hospital, blood tested suitable for transfusion is more available during periods of blood shortage than in peripheral hospitals. Hemoglobinuria followed with $38.1 \%$, as also reported in Togo in 2002 [7]. By contrast, in a study conducted in Senegal in 2007, its occurrence was not as regular [8]. It would be interesting to investigate (through a scientific study) the reasons for the high frequency of hemoglobinuria compared to other countries. Among the children, 87 (19.4\%) had bacterial co-infection. It is assumed that passage of hematozoa 
into the blood may induce immunosuppression, albeit moderate and temporary [15].

\subsection{Therapeutic Aspects and Outcomes}

In this investigative work, artesunate was the molecule most used, as recommended by the WHO [1], except for 4 children who were administered quinine. The average length of stay in hospital was 5 days (range, 0 to 25 days). In this study, in terms of severe malaria, 59 children experienced fatal outcomes (i.e., a hospital-related lethality rate estimated at $13.1 \%$, IC: $10.0 \%-16.3 \%)$. These figures are close to those reported in two other studies performed in Sub-Saharan Africa [12] [16]. Overall, severe malaria continues to exact a heavy toll on children. In 2015, the United Nations Inter-Agency Group for Child Mortality Estimation (UN-IGME) reported malaria as the third preventable cause of death in children under 5 years of age, after pneumonia and diarrhea [17], despite the fact that a practical handbook for the management of malaria has been edited by the WHO [1]. Two of three children died within the first 24 hours of hospitalization. Another researcher has made the same remark [5]. This report is about early deaths that reflect the particularly serious condition of children at admission. Late use of medical care due to initial lack of knowledge of serious symptoms by parents may explain the early deaths.

\subsection{Factors Associated with Death}

Death occurred without differentiation in both sexes. Other authors have reported that female children had a greater risk of death [5] [18].

The univariate analysis results showed that the risk of mortality was significantly associated with coma $(\mathrm{OR}=4.78)$, respiratory distress $(\mathrm{OR}=3.62)$, convulsions $(\mathrm{OR}=2.19)$, hypoglycemia $(\mathrm{OR}=4.64)$ and bacterial co-infection ( $\mathrm{OR}$ $=2.25)$, particularly septicemia $(\mathrm{OR}=10.31)$. According to a study conducted in Congo in 2011, the factors identified as associated with death were hypovolemic shock, coma and association of at least two serious symptoms [10]. In Ghana [12], in addition to the factors mentioned above, other factors were identified as being associated with death (e.g., hyperparasitemia and hyperlactatemia). Coma was one of the serious symptoms mostly observed among dead children (59\%). The neurological forms of severe malaria, including coma, are still a leading cause of child mortality, despite a better understanding of pathophysiological mechanisms and recommendations related to care. The results noted in the study confirm the frequency and prognostic value of respiratory distress. The relevance of that factor in children has been emphasized in other African studies [10] [18]. Respiratory distress was associated with acidosis, anemia and acute pulmonary edema. Indeed, pulmonary edema is a serious complication of severe Plasmodium falciparum malaria, with high mortality rate (greater than $80 \%$ ). Unfortunately, we did not have the opportunity to analyze blood gases in this study. There was a statistically significant relationship between hypoglycemia and death occurrence. Hypoglyce- 
mia is the outcome of increased metabolic needs and limited reserves of glycogen, particularly in children under 3 years of age. Moreover, clinically, it is easily overlooked, as its manifestations may be similar to ones of cerebral malaria [1]. This research has demonstrated a relationship between convulsions and death. There were repeated convulsions often indicating a convulsive status epilepticus likely associated with brain edema. Brain edema results from sequestration of $P$. falciparum in the capillaries of the brain on the one hand, and from production of inflammatory cytokines on the other hand. Thus, brain edema formed would worsen ischemia by inducing compression of the arteries, which might be responsible for the reduced cerebral blood flow causing brain injury (neurological damage) and even death [19]. In the context of our work, sub-optimal intensive care measures are all factors that contributed to the poor prognosis observed in this study in cases of convulsive status epilepticus or coma with brain edema.

Bacterial co-infection, particularly septicemia, was a critical factor in the occurrence of death in children admitted for malaria (with a mortality risk 10 times higher). Septicemia is a severe bacterial infection that is life-threatening, unlike pneumonia which may be more easily treated. The kinds of bacteria were gram negative.

\section{Conclusion}

In this study of the factors related to fatal outcomes in cases of severe malaria among children hospitalized in the CNHU-HKM's pediatric and medical genetics clinic, the malaria mortality rate was $13.1 \%$. In addition, the factors identified as being critical in the occurrence of child death were coma, convulsive status epilepticus and bacterial co-infection. Corrective measures should be put in place to curb these identified factors to reduce the lethality associated with severe malaria.

\section{References}

[1] World Health Organization (WHO) (2013) Management of Severe Malaria-A Practical Handbook. 3rd Edition, WHO, Geneva.

[2] World Health Organization (2014) The African Malaria Report, Tech. Report WHO/CDS/ MAL/2014, 1093, Geneva.

[3] Aubry, P. and Gauzère, B. (2015-2016) An Update on Malaria. Medecine Tropicale, 1-26.

[4] Robert, W. (2015) Global Malaria Eradication and the Importance of Plasmodium falciparum Epidemiology in Africa. Snow BMC Medecine, 13, 23.

[5] Muoneke, V.U., Ibekwe, R.C., Neb-Agumadu, H.U., et al. (2012) Factors Associated with Mortality in Under-Five Children with Severe Anemia in Ebonyi, Nigeria. Indian Pediatrics, 119, 119-123. https://doi.org/10.1007/s13312-012-0026-4

[6] Kalinga, A., Mayige, M., Kagaruki, G., et al. (2012) Clinical Manifestations and Outcomes of Severe Malaria among Children Admitted to Rungwe and Kyela District Hospitals in South-Western Tanzania. Tanzania Journal of Health Research, 14, 1-9. https://doi.org/10.4314/thrb.v14i1.2

[7] Gbadoé, A.D., Kini-Caussi, M., Koffi, S., et al. (2006) Outcome of Severe Pediatric Malaria in Togo between 2000 and 2002. Médecine et Maladies Infectieuses, 36, 52-54. 
https://doi.org/10.1016/j.medmal.2005.10.006

[8] Camara, B., Diagne-Gueye, N.R., Faye, P.M., et al. (2011) Severity Criteria and Prognostic Factors of Malaria among Children in Dakar. Médecine et maladies infectieuses, 41, 63-67. https://doi.org/10.1016/j.medmal.2010.09.001

[9] Camara, B., Diouf, S., Diagne, L., et al. (2003) Severe Malaria among Children in a Senegal Hospital Setting. Médecine et maladies infectieuses, 33, 45-48. https://doi.org/10.1016/S0399-077X(02)00014-8

[10] Liosimba, L.J., D’Alessandro, U., Donnen, P., et al. (2012) Clinical Aspects and Outcome of Suspected Severe Pediatric Malaria. Médecine et maladies infectieuses, 42, 315-320. https://doi.org/10.1016/j.medmal.2012.05.008

[11] Pasvol, G., Weatherall, D.J. and Wilsonn, R.J.M. (1977) Effects of Foetal Haemoglobin on Susceptibility of Red Cells to Plasmodium falciparum. Nature, 270, 171-173. https://doi.org/10.1038/270171a0

[12] Mokenhaupt, F.P., Ehrhardt, S., Burkhardt, J., et al. (2004) Manifestation and Outcome of Severe Malaria in Children in Northern Ghana. The American Journal of Tropical Medicine and Hygiene, 71, 167-172.

[13] Yip, R. (1996) Prevention and Control of Iron Deficiency in Developing Countries. Current Issues in Public Health, 2, 253-263.

[14] World Health Organization (2000) Management of Severe Malaria. A Practical Handbook, Vol. 2, Annex 3.

[15] Ammah, A., Nkuo-Akenji, T., Ndip, R., et al. (1999) An Update on Concurrent Malaria and Typhoid Fever in Cameroon. Transactions of the Royal Society of Tropical Medicine and Hygiene, 93, 127-129. https://doi.org/10.1016/S0035-9203(99)90282-1

[16] Mabiala-Babela, J.R., Ollandzobo, L.C., Ikoboa, E.R., et al. (2015) Clinical Profile and Outcome of Severe Anemia Due to Malaria in Congolese Children. Pediatric Archives, 22, 323-328.

[17] UN Inter-Agency Group for Child Mortality Estimation (UN-IGME) (2015) Levels and Trends in Child Mortality. Report.

[18] Dzeing-Ella, A., Nze Obiang, P.C., Tchoua, R., et al. (2005) Severe Falciparum Malaria in Gabonese Children: Clinical and Laboratory Features. Malaria Journal, 4, 8 p.

[19] Ilunga-Ilunga, F., Levêque, A., Donnen, P., et al. (2015) Characteristics of Households of Children Hospitalized for Severe Malaria and Factors Associated with Malaria Lethality in Kinshasa (Democratic Republic of Congo). Medecine et Sante Tropicales, 25, 75-81. 